Validation of the virtual elevation field test method when assessing the aerodynamics of para-cyclists with a uni-lateral trans-tibial amputation.

Bryce Dyer $^{1 *}$, B Xavier Disley²

${ }^{1}$ Faculty of Science \& Technology, Bournemouth University, Poole, UK*.

brdyer@bournemouth.ac.uk

${ }_{2}^{2}$ Aerocoach Ltd, Bromsgrove, UK. xavier@aero-coach.co.uk

*Corresponding author 


\title{
Validation of the virtual elevation field test method when assessing the aerodynamics of cyclists with a uni-lateral trans-tibial amputation.
}

\author{
Lower-limb amputees typically require some form of prosthetic limb to ride a \\ bicycle for recreation or when competing. At elite-level racing speeds, \\ aerodynamic drag can represent the majority of the resistance acting against a \\ cyclists forward motion. As a result, the reduction of such resistance is beneficial \\ to an amputee whereby the form and function of the prosthetic limb can be \\ optimised through engineering. To measure the performance of such limbs, field \\ testing provides a cost effective and context specific method of aerodynamic drag \\ measurement. However, few methods have been formally validated and none \\ have been applied to amputees with lower-limb amputations. In this paper, an \\ elite level para-cyclist wore two different prosthetic limb designs and had their \\ total aerodynamic drag of a wind tunnel reference method statistically correlated \\ against a velodrome-based virtual elevation field test method. The calculated \\ coefficient of variation was in the range of $0.7-0.9 \%$ for the wind tunnel method \\ and $2-3 \%$ for the virtual elevation method. A $0.03 \mathrm{~m}^{2}$ difference was identified in \\ the absolute values recorded between the two methods. Ultimately, both methods \\ exhibited high levels of precision, yet relative results to each other. The virtual \\ elevation method is proposed as a suitable technique to assess the aerodynamic \\ drag of amputee para-cyclists.
}

Keywords: Prosthesis; amputation; cycling; aerodynamics

\section{Background}

The opportunities for individuals with an amputated limb using cycling as a medium for physical activity have developed from one of purely recreation [1] into more extreme pursuits such as competitive cycling [2] or triathlon [3]. However, to undertake cycling whilst possessing any level of lower-limb amputation will likely require some form of specialised prostheses to be developed [4]. Furthermore, the ability to optimise the performance of such individuals undertaking competitive cycling is desirable for 
sporting events such as the Paralympic Games. However, a recent systematic review revealed that there is extremely limited peer-reviewed literature to help inform the clinicians with the design of either adaptive or assistive technology for this purpose [2].

The role of aerodynamics plays a vital role in competitive cycling [5]. At elitelevel racing speeds, aerodynamic drag can represent upto $96 \%$ of the cyclist's power [6]. It has also been found that $31-39 \%$ of the wind resistance or drag is due to the bicycle equipment [7]. It is suggested that changes in a cyclists equipment or their riding position can alter their frontal area and therefore the net aerodynamic drag [8]. This could ultimately lead to substantial performance improvements[8]. At the very least, it has been proposed that the aerodynamics of a lower-limb prosthesis could be made advantageous over the equivalent biological limb region [9]. Nonetheless, whilst various methods have been proposed to evaluate the aerodynamic properties of cyclists and/or their equipment [5], only two studies have focused on an athlete with a lowerlimb amputation [9][10].

In able-bodied cycling, the use of a wind tunnel has been successfully validated and used as a source of cross-method validation of aerodynamic drag [11] [12]. However, wind tunnels are often geographically few in number and prohibitively expensive. Alternatively, field testing has also been proposed as a more affordable method of assessment which only requires a power meter device fitted to the bicycle and an environment conducive to such testing [13]. These environments typically comprise a flat surface that is undisturbed by traffic, such as an aircraft runway or a cycling velodrome [11] [12]. To date, outdoor field testing has also been demonstrated to be statistically robust [5] and successfully correlated to wind tunnel testing of able-bodied riders [11] [12]. Alternatively, the Virtual Elevation method (VE) is another means of aerodynamic drag field-testing [14]. This method 
has not yet been validated in any peer reviewed research. In addition, no study to date has aerodynamically assessed a competitive cyclist with a lower-limb amputation in a field test or a wind tunnel environment. In this paper, the VE method is investigated to help address both of these gaps in knowledge and is validated against a wind tunnel reference method.

\section{Methods}

A male, below-knee unilateral amputee acted as the participant for this study. The use of a single participant has been indicated as typical of many studies involving competitive para-cyclists [2].

The participant was an international standard track and road para-cyclist with a uni-lateral trans-tibial amputation of their right limb. The participant was aged 33, had a height of $1.72 \mathrm{~m}$, a weight of $73 \mathrm{~kg}$ and had personal best cycling performances including 21 minutes and 31 seconds for a $16.1 \mathrm{~km}$ individual time trial and 3 minutes and 56 seconds for the $3 \mathrm{~km}$ individual track pursuit. These trials would assess the total aerodynamic drag obtained in the wind tunnel (WT) when using two different leg prostheses. It would then compare these to the calculated aerodynamic drag using the Virtual Elevation (VE) field test method when using the same participant. The two prostheses were identical in their overall length, pedal cleat position and socket design. The pylon region began $80 \mathrm{~mm}$ up from the base of the foot and was $270 \mathrm{~mm}$ long. This specific area formed the key difference between the two prostheses. Only the depth and profile of the pylon region itself was altered. One prostheses possessed a pylon region that was $18 \mathrm{~mm}$ in diameter and round in section (prosthesis A). The other prostheses possessed a pylon region that was aerofoil shaped and was $18 \mathrm{~mm}$ in width, and $108 \mathrm{~mm}$ in depth (prosthesis 
B). Whilst the appearance of a prosthetic limb could assume to be standardised, in reality it is typical for the socket fit, prostheses biomechanics and overall form itself to require differences from user to user.

The participant used the same prosthetic liner when wearing all of the test prostheses. The liner was an Ossur Seal-in X5 (Ossur hf, Reykjavik, Iceland) coupled with an Ossur L-551002 valve (Ossur hf, Reykjavik, Iceland) to provide the suction suspension. The participant provided informed consent and the author obtained institutional ethics approval for this study.

\section{Wind Tunnel Test Protocol}

The wind tunnel data collection was carried out using a closed-circuit wind tunnel (Southampton University, Southampton, UK). Measurements were undertaken at air speeds of $14 \mathrm{~m} / \mathrm{s}$ and $16 \mathrm{~m} / \mathrm{s}$. These are representative of elite-level, short distance velodrome-based track cycling events such as the time trial (performed over either $500 \mathrm{~m}$ or $1 \mathrm{~km}$ ) and the individual pursuit events (performed over either $3 \mathrm{~km}$ or $4 \mathrm{~km})$. Bassett et al. [15] estimated that cyclists' drag coefficient is typically constant when wind speed ranges between 50 and $60 \mathrm{~km} / \mathrm{h}$ and this was representative of those used in these trials. Three wind yaw angles (i.e. the angle of alignment between the rider and bicycle to that of the oncoming air flow) were used in 5 degree increments as per Martin et al. [11] and were specified as 0, 5 and 10 degrees. This angle was achieved by rotating the bicycle which was positioned on a turntable and was an established fixture of the wind tunnel environment. These yaw measurements are similar to the limited evidence that exists to the actual yaw angles experienced by a cyclist riding on a velodrome and these were defined as a range of 3-10 degrees [16, p.31]. Therefore, the overall 
performance of each change in prosthetic limb was taken as a mean average of the three yaw angles and the mean average of both the $14 \mathrm{~m} / \mathrm{s}$ and $16 \mathrm{~m} / \mathrm{s}$ test speeds.

The aerodynamic drag was measured using a force transducer with a precision of $+/-0.5 \%$ and an air flow precision of $+/-0.02 \mathrm{~m} / \mathrm{s}$. The data was sampled at $97 \mathrm{~Hz}$ for 20 seconds and had three runs performed at each wind speed per prosthetic leg. This method was repeated for both of the tested yaw angles. The WT area was $8.174 \mathrm{~m}^{2}$ and when assuming a frontal area of $0.35 \mathrm{~m}^{2}$, the blockage would be considered less $<5 \%$.

The bicycle was mounted at the front and rear wheel axle points and positioned on top of rollers that were underneath each wheel. The front and rear rollers were connected by a drive band. This ensured that both of the wheels rotated at the same velocity when the participant turned the cranks. An image of the wind tunnel rig is shown in figure 1.

\section{[FIGURE 1 HERE]}

\section{The Virtual Elevation Method Protocol}

The participants bicycle, helmet, clothing and their overall riding position were unchanged from the WT data collection phase. Whilst there are other validated aerodynamic field test methods [17], VE does not require the need for a constant power output, elevation or riding speed and has been successfully applied to the assessment of competitive cycling efforts [14]. However, there is no application of this technique to date within peer reviewed literature. In addition, its ability to be utilised in either velodromes or outdoor spaces would likely be of lower cost when compared to commercial installations such as a wind tunnel. 
The VE method records a moment-by-moment speed and power output from a lap (or laps) ridden over a known route. From this, it constructs an elevation profile for the ride as a function of known power, speed, mass and air density using initial guesses of aerodynamic drag (CdA) and the coefficient of rolling resistance (Crr). Since each lap would start and end at the same point, the CdA value is altered until it produces a zero net gain in elevation over each lap. By achieving this, the assumed CdA value (and therefore the aerodynamic drag) is perceived as correct.

The power (in Watts) needed to propel a rider at speed $v$ is the total of the power to overcome rolling resistance, the power to account for the change in elevation (the potential energy), the power to account for a change in speed (the kinetic energy) and the power to account for air resistance. This formula was defined by Chung [14] as:

$P=\mathrm{C}_{\mathrm{rr}} m g v_{\text {ground }}+s m g v_{\text {ground }}+m a v_{\text {ground }}+1 / 2 C d A \rho v_{\text {air }}^{2} v_{\text {ground }}$

Where:

$P=$ Total Power

$\mathrm{C}_{\mathrm{rr}}=$ Coefficient of rolling resistance

$m=$ total mass $(\mathrm{Kg})$ of rider and bicycle

$g=9.81 \mathrm{~m} / \mathrm{sec}^{2}$

$v_{\text {ground }}=$ ground speed $(\mathrm{m} / \mathrm{s})$

$s$ = slope

$a=$ acceleration

$\rho=$ air density

$v_{\text {air }}=$ air speed of the bike 
$C d A=$ drag area of the rider and bicycle

This equation makes an assumption that the ground speed and air speed are the same. As a result, this means that any field test using this method must be undertaken when a low or (preferably) no additional wind is present otherwise error will be introduced into the calculations. This was the rationale for using an indoor velodrome in this study as a testing environment as it was an enclosed facility.

This equation can then be solved for slope (s) which Chung [14] then defines as:

$s=P /\left(m g v_{\text {ground }}\right)-C_{r r}-a / g-\left(\rho C d A v_{\text {ground }}\right) /(2 m g)$

This equation was used iteratively with both the velocity and the distance recorded to create an elevation profile of each lap ridden.

The Crr value was ascertained from previous trials and was defined as 0.004 . The total mass of rider and bike was $86 \mathrm{Kg}$. The power output of the rider was then captured using a ‘Powertap’ rear wheel hub-based power meter (Powertap, Madison, WI, USA) contained within a disc wheel. This has a manufacturer's proposed accuracy of $+/-1.5 \%$. This power meter has been validated for use in cycling related studies before [18] and has been corroborated against other cycling power measurement systems [19]. The sampling rate of this device was $100 \mathrm{~Hz}$ and a wireless ANT+ protocol was used to transmit a sampling rate of $1 \mathrm{~Hz}$ to a Garmin 500 wireless GPS unit (Garmin, Kansas, US) . The virtual elevation data was obtained using a Garmin speed sensor mounted to the bicycle and then also transmitted to the same wireless unit. All devices were zeroed according to the manufacturer's instructions prior to the tests commencing. 
The aerodynamic field tests took place on an indoor 250m banked velodrome (Newport, Wales, UK) with a wooden surface. This type of environment has been validated for aerodynamic testing [12]. Weather data was taken from the nearest weather station to calculate the air density. The air density value was derived from a calculator provided from a previous study by Martin et al. [17]. The air temperature was $22^{\circ} \mathrm{C}$, the dew point $10^{\circ} \mathrm{C}$, the pressure was $1016 \mathrm{mb}$. These measurements produced a calculated air density (Rho) of 1.198. The participant rode their own time trial bicycle on the velodrome track and wore the same clothing and helmet for all of the trials. No other riders were present on the track at any time during the testing so as to avoid creating any unwanted additional air flow or turbulence.

The testing protocol required the participant to fit the selected test prosthesis at the velodrome trackside. They then mounted their bicycle and undertook a test run that involved 20 laps of the velodrome. This meant each test run equated to a riding distance of 5km. During each 5km trial, the participant cycled at three predetermined and physically repeatable targeted speeds of $42 \mathrm{~km} / \mathrm{h}, 38 \mathrm{~km} / \mathrm{h}$ and $34 \mathrm{~km} / \mathrm{h}$. A range of different riding velocities have been recommended for aerodynamic drag field testing [17]. These particular speeds are typical of those performed in racing with a disability but were also felt to be sustainable by the participant over the full length of the experiment when riding on a velodrome. Each 5km run was broken down as 6 laps targeted at $42 \mathrm{~km} / \mathrm{h}$ followed by 1 lap to slow down and establish a new cruising speed. This was followed by another 6 laps at $38 \mathrm{~km} / \mathrm{h}$ followed by another lap to slow down and establish the next targeted steady state cruise speed. Finally, there were 6 laps performed at $34 \mathrm{~km} / \mathrm{h}$. After this had been completed, the rider slowed down, dismounted the bicycle and then swapped the test prosthesis for another before then resuming the next $5 \mathrm{~km}$ test run. Two test runs of each leg design were performed and 
the two prostheses were alternated. This meant that each leg received 6 separate test intervals.

\section{Statistical Analysis}

Microsoft Excel was used for the statistical analysis of the data. The outcomes of the changes in prosthetic limbs were reported as means with a calculated standard error for each of the three yaw angles and the overall mean average performance. Additionally, the Coefficient of Variation (CV) was then also calculated and expressed as a percentage for the results of each prosthetic limb when using both methods to ascertain the level of test method data variability. The differences in the overall mean aerodynamic drag for each prostheses used (which included both wind speeds and the three test yaws) were determined by a single factor ANOVA and supported by paired Student's $t$-tests between the two different prosthetic leg changes. The level of statistical significance was set as $\mathrm{p}=<0.05$.

\section{Results}

A comparison of the aerodynamic drag $\left(\mathrm{m}^{2}\right)$, standard error and coefficient of variation (\%) produced by the participant from both the WT and the VE methods using the two prostheses designs are shown in table 1.

\section{[TABLE 1 HERE]}

The CV of the two changes in prosthetic limbs were in a range of $0.7-0.9 \%$ from the WT tests and 2-3\% from the VE tests. These are low values thereby demonstrating that both methods achieved a high level of data precision of their calculated aerodynamic 
drag. However, the WT method achieved a lower level of standard error and CV when compared to the VE method when considering each yaw angle separately. Prostheses B achieved the lowest value of overall aerodynamic drag. The mean average of the overall aerodynamic drag of each leg prostheses change when using the WT method compared to the VE method is shown in table 2 .

[TABLE 2 HERE]

The use of ANOVA demonstrated that the overall aerodynamic drag performance of the two prostheses in the VE tests were not statistically significant from each other ( $p=>0.05$ ) with the subsequent $t$-tests revealing that this characteristic was consistent between both prostheses designs ( $p=>0.05$ ). However, the WT results between the two prostheses designs did demonstrate statistical significance between them when performing $t$-tests between the 0 and 5 degrees yaw results $(p=<0.05)$ but not between 0 and 10 degrees or between 5 and 10 degrees results ( $p=>0.05)$. In essence, the performance of prostheses B over A reduced at the widest yaw angle evaluated.

\section{Discussion}

Both methods demonstrated a very high level of test run repeatability which was exhibited by the low CV values. In addition, the VE method obtained a similar level of CV (2.1\%) to another published aerodynamic field test method that was also intended for prosthetic level changes in componentry [10]. This suggests that despite limb to limb asymmetry being a typical characteristic of cyclists with lower-limb absence [2], the very high level of correlation and low data variability in this study suggests that 
either test is recommended for the analysis of cyclists that possess a lower-limb amputation.

The ANOVA and $t$-tests revealed that the impact of changing prosthetic limb did not produce a significant difference in the overall aerodynamic drag of the participant when using the VE method. However, any change in cycling technology could ultimately produce marginal, yet positive improvements. It was still felt that the measured difference in aerodynamic drag between the two prostheses (coupled with their small standard error and low CV values from both test methods) provided further evidence that optimised prosthetic limb design would impact positively on the performance of cyclists with lower-limb amputations. This supports the findings indicated in previous studies [9] [10].

The drag measurement values obtained between the two methods were distinctly different from each other. When WT-based testing has been assumed to be the superior or reference method, velodrome-based testing has been shown to underestimate the aerodynamic drag of a cyclist [12]. With both methods in this paper recording low standard error and data variability, it is suggested that a difference in the test methodology’s may well be responsible for the differences in their calculated aerodynamic drag. There may be several reasons that might contribute to this discrepancy. One might be the impact of bicycle crank cadence influenced aerodynamic drag. Whilst the rider turns the cranks in both methods, the cadence could not be recorded using the WT protocol and was self-selected by the participant. In addition, the influence of a velodrome possessing two tight bend turns per lap would likely not produce the same steady-state cadence as the wind tunnel environment. This could mean that the differences in aerodynamic drag may have been affected by any difference in crank cadence and any resulting aerodynamic interference effects from the 
prostheses and the bicycle itself. Another reason might be that the wind yaw measurements that were taken were achieved by rotating the rider anti clockwise on a turntable (meaning the wind was striking the drivetrain side of the bicycle). However, a wind tunnel capable of yaw angles performed on both sides of the rider may shed further light on this discrepancy. For example, it was revealed that the bicycle used by Team USA at the 2016 Olympic Games for racing on the velodrome unusually had its drivetrain on the left hand side of the bicycle. It has been suggested that this would reduce a rider's aerodynamic drag when riding on a velodrome. Whilst the margin of this development has not been measured scientifically, this may well be a consideration to riders with lower-limb amputations who wish to accurately determine their aerodynamic drag on a velodrome. It could also be argued that the yaw angles experienced by the participant would not always have been proportionally identical between both test methods. There is a paucity of published yaw angle data when riding a bicycle on a velodrome to be in a position to align these exactly. In addition, it was seen in this study that the calculated aerodynamic drag is affected by the resulting wind yaw angle that a cyclist is being subjected to. As a result, it is entirely plausible that such a discrepancy may have occurred due to the specific conditions of velodrome cycling. Whilst the limited data that does exist suggests that yaws of 10 degrees have been recorded when riding the bends of a velodrome (Burke, 2003, p31), it should be noted that the difference in the overall results between the two methods would have reduced significantly if the 10 degree yaw data were discounted. This would have reduced the discrepancy between the two methods by $0.012-0.014 \mathrm{~m}^{2}$. As a result, there is a need to investigate the resulting wind yaw angles when riding an indoor velodrome at a para-cyclists competitive velocity. It is also possible that any biomechanical asymmetry of such cyclists when in a wind tunnel might also provide some basis for 
any statistical discrepancy. For example, since the bicycle in the wind tunnel is fixed, an athlete with a uni-lateral amputation may have exhibited more asymmetrical body movement that may not have been evident when riding freely. This study was the first investigation of such athletes. As a result, cyclists with and without limb absence should be directly compared in the future to ascertain what general differences may exist. Likewise, it is also recommended that the assessment of the VE method should be expanded to include larger or more diverse sample groups as well as different test environments.

Despite the unknown root cause of the difference in measured CdA between the two methods, VE is recommended as a statistically precise method when assessing the aerodynamic drag of cyclists with a lower-limb amputation.

\section{Conclusion}

The virtual elevation method was used to evaluate the aerodynamic drag of a cyclist with a lower-limb amputation using two different prosthetic limbs. These results were compared to the use of a wind tunnel reference method. A high level of statistical correlation was produced between the two methods. In addition, both methods produced a high degree of statistical precision. However, the virtual elevation method produced lower aerodynamic drag values when compared to the wind tunnel reference method. This makes the results relative to each other and not directly interchangeable. The virtual elevation method is recommended for evaluating the aerodynamic drag of cyclists who require the use of a prosthetic limb.

\section{Acknowledgements}


The authors wish to thank the cyclist that participated in this study. The authors would also like to thank Pace Rehabilitation Ltd. for the manufacture and supply of the prosthetic limbs used for this study.

Word Count: 3654

\section{References}

[1] Legro M, Reiber G, Czerniecki J, Sangeorzan B. Recreational activities of lowerlimb amputees with prostheses. Journal of Rehabilitation Research and Development. 2001;38:319-325.

[2] Dyer B. Cycling with an amputation: a systematic review. Prosthetics and Orthotics International. 2016. DOI:0309364615610659.

[3] Gailey R, Harsch P. Introduction to triathlon for the lower limb amputee triathlete. Prosthetics and Orthotics International. 2009;33:242-255.

[4] Michael J, Gailey R, Bowker J. New developments in recreational prostheses and adaptive devices for the amputee. Clinical Orthopaedics and Related Research. 1990;256:64-75.

[5] Debraux P, Grappe F, Manolova A, Bertucci W. Aerodynamic drag in cycling: methods of assessment. Sports Biomechanics. 2011;10:197-218.

[6] Martin J, Gardner S, Barras M, Martin D. Modelling sprint cycling using fieldderived parameters and forward integration. Medicine \& Sport in Sports \& Exercise. 2006;38:592-597.

[7] Kyle C, Burke E. Improving the racing bicycle. Mechanical Engineering. 1984;9: 34-45. 
[8] Keogh J. Paralympic sport: an emerging area for research and consultancy in sports biomechanics. Sports Biomechanics. 2011;10:234-253.

[9] Childers W, Gallagher T, Duncan J, Taylor D. Modeling the effect of a prosthetic limb on 4-km pursuit performance. International Journal of Sports Physiology \& Performance 2015;10:3-10.

[10] Dyer B. The importance of aerodynamics for prosthetic limb design used by comeptitive cyclists with an amputation: an introduction. Prosthetics and Orthotics International. 2014;39:232-237.

[11] Martin J, Milliken D, Cobb J, McFadden K, Coggan, A. Validation of a mathematical model for road cycling power. Journal of Applied Biomechanics. 1998;14:276-291.

[12] Garcia-Lopez J, Alday-Ogueta A, Larrazabal J, Rodriguez-Marroyo A. The use of velodrome tests to evaluate aerodynamic drag in professional cyclists. International Journal of Sports Medicine. 2013; doi.org/10.1055/s-0033-1355352.

[13] Lim A, Homestead E, Edwards A, Carver T, Kram R, Barnes W. Measuring changes in aerodynamic/rolling resistances by cycle mounted power meters. Medicine \& Science in Sport \& Exercise. 2011;44:853-860.

[14] Chung R. Estimating CdA with a power meter. 2012; http://anonymous.coward.free.fr/wattage/cda/indirect-cda.pdf. Accessed 5 Sept 2015. [15] Bassett D, Kyle C, Passfield L, Broker J, Burke E. Comparing cycling world hour records, 1967 - 1996: modelling with empirical data. Medicine and Science in Sports and Exercise. 1999;31:1665 - 1676.

[16] Burke E. 2003. High-tech cycling. Human Kinetics.

[17] Martin J, Gardner S, Barras M, Martin D. Aerodynamic drag area of cyclists determined with field-based measures. Sportscience. 2006;10:68-69. 
[18] Bertucci W, Duc S, Villerius V, Pernin J, Grappe F. Validity and reliability of the PowerTap mobile cycling powermeter when compared with the SRM device. International Journal of Sports Medicine. 2005;26:868-873.

[19] Gardner A, Stephens S, Martin D, Lawton E, Lee H, Jenkins D. Accuracy of SRM and power tap power monitoring systems for bicycling. Medicine and Science in Sports and Exercise. 2004;36:1252-1258. 